\title{
Chapter 15 \\ Monitoring Systems \\ of an Electrospinning Plant \\ for the Production of Composite \\ Nanofibers
}

\author{
Luca Bonura, Giacomo Bianchi, Diego Omar Sanchez Ramirez, \\ Riccardo Andrea Carletto, Alessio Varesano, Claudia Vineis, \\ Cinzia Tonetti, Giorgio Mazzuchetti, Ettore Lanzarone, \\ Simona Ortelli, Anna Luisa Costa and Magda Blosi
}

\begin{abstract}
Electrospinning is a versatile and promising technology for the production of polymer-based nanofibres. Composite nanofibres suitable for filtration of air and water have been developed by merging biopolymer processing and sol-gel techniques using electrospinning technology. A fine control of large-scale nanofibre formation is required to achieve reliable transfer of electrospinning-based processes into relevant industrial environments. The main goals of this work were the production of innovative multifunctional filter media (high-efficiency filtration, biocidal, self-cleaning, photo-catalytic, reactive, adsorbent properties) by integrating nanofibre membranes with inorganic nanoparticles and developing an electrospinning plant integrating sensors able to detect electrospinning fault and electrostatic alteration during the process.
\end{abstract}

\footnotetext{
L. Bonura $\cdot$ G. Bianchi

CNR-STIIMA, Istituto di Sistemi e Tecnologie Industriali Intelligenti per il Manifatturiero Avanzato, Milan, Italy

D. O. Sanchez Ramirez · R. A. Carletto · A. Varesano $(\bowtie) \cdot$ C. Vineis · C. Tonetti · G. Mazzuchetti CNR-ISMAC, Istituto per lo Studio delle Macromolecole, Biella, Italy

e-mail: a.varesano@bi.ismac.cnr.it

E. Lanzarone

CNR-IMATI, Istituto di Matematica Applicata e Tecnologie Informatiche, Milan, Italy

S. Ortelli $\cdot$ A. L. Costa $\cdot$ M. Blosi

CNR-ISTEC, Istituto di scienza e tecnologia dei materiali ceramici, Faenza, Ravenna, Italy

(C) The Author(s) 2019

T. Tolio et al. (eds.), Factories of the Future,

https://doi.org/10.1007/978-3-319-94358-9_15
} 


\subsection{Scientific and Industrial Motivations}

Non-wovens are textile materials consisting of randomly oriented fibres linked together by bonds or physical entanglements between fibres, without any knitting or stitching. These textile materials are usually produced by putting staple fibres together to create a web and then binding them thermally (wet-laid, air-laid, carding) or mechanically or by spinning a molten polymer into continuous filaments directly distributed into a web by air streams and deflectors (melt-blown, spun-bond). Nonwovens are the primary alternative to traditional textiles as filtration media, thermal and sound insulating materials, hygienic and health/personal care textiles, automotive textiles, building materials and geo-textiles [1].

Emerging applications require fibres with decreased sizes. Since the specific surface area is proportional to the diameter of the fibre and the solid volume is proportional to the square of the diameter, the specific surface area is inversely proportional to the diameter of the fibre, thus leading to great surface area for small fibres. Moreover, apparent size of pores also depends on the diameter of the fibre, thus thin fibres produce non-wovens with small pores able to entrap and remove fine particles (penetrating particles) [1].

There are several methods for producing thin fibres with high-volume production methods like island-in-sea, fibrillation and innovative melt-blowing system or highly accurate methods such as nanolithography and self-assembly. However, combinations of a limited number of materials, elevated costs and low production rates restrict their usefulness. Instead, electrospinning is a simple and low cost process characterised an intermediate throughput. Electrospinning is an electro-hydrodynamic process that is generally considered the most promising and adaptable platform technology for the manufacturing of nanofibres. Briefly, the process takes place between a metal capillary opening (where a high voltage is applied), and a static (usually grounded) collector acting as a nanofibre gathering counter-electrode. The metal capillary is connected to a reservoir feeding the polymer solution under pressure (Fig. 15.1). The high voltage between the capillary and the grounded collector results in a nano- to micron-sized electrically-driven stream of polymer solution which is drawn out from the apex of a cone-shaped droplet (so-called Taylor cone) formed at the electrically-charged capillary opening. The solvent evaporates from the jet during the flight and, under ideal conditions, a continuous nano-sized filament is gathered on the collector in a random mode forming a 2D isotropic structure. Electrospun nanofibres are unique compared to other conventional materials due to their high surface to volume ratio, high fibre interconnectivity and nano-scale interstitial spaces. Nanofibres are therefore of considerable interest in applications where structures with a high surface area are desirable (e.g., filtration, biomedicine, composite materials). Emerging applications of electrospun nanofibres are in the field of textiles, as high-efficiency filters for air and liquids.

The first objective of the work was to produce innovative multifunctional filter media by integrating keratin nanofibre membranes with inorganic nanoparticles (as nanometals or nanometaloxides) by electrospinning. The advantage of keratin-based 


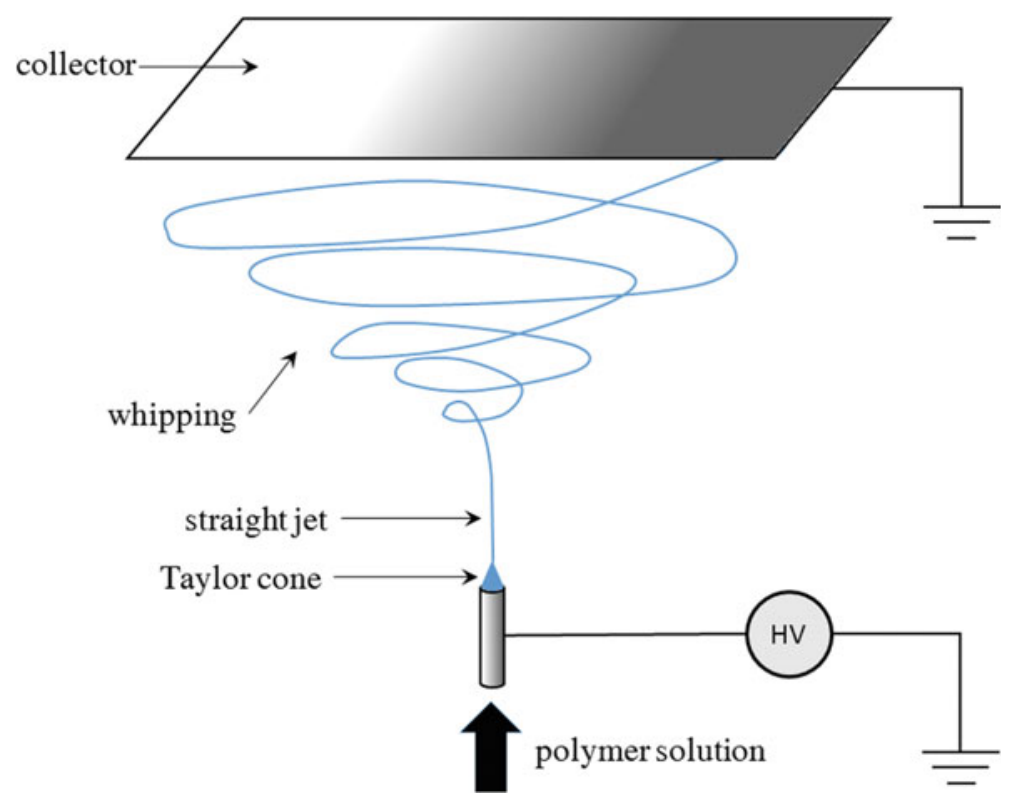

Fig. 15.1 Scheme of the electrospinning process

nanofibre membranes is that they offer both removal of suspended small particles filtration (due to the controlled porosity and small pore size) and adsorption of heavymetals, dyes and VOCs, such as formaldehyde (due to the presence of side-chain functional groups able to chemically bind harmful chemicals) [2-6]. Nano-metals and/or nano-metal oxides improve membrane functionalities related to kill of bacteria (antimicrobial, low bio-fouling) and photocatalytic properties (removal of organic chemical compounds), respectively [7-12]. The results demonstrated that embedding inorganic nanoparticles onto keratin nanofibres makes possible to produce nanoreactive filters active for selective removal of pollutants in air and water treatments [13]. For the realization of nano-reactive filters it is necessary to prepare stable and electrospinnable keratin solutions doped with inorganic nanoparticles. In particular, keratin/Ag and $\mathrm{TiO}_{2}$ ceramic nanoparticles dispersions were prepared by mixing keratin solutions and colloid dispersions.

Electrospinning is a technology currently at the border between basic research and industrial implementation. Recently, large-scale electrospinning systems have been developed by different research groups [14-21] in order to increase the productivity of the process at industrial level. Currently, the electrospinning process is mostly running with the operator setting the voltage and feeding flow by an open loop approach. An advanced control of nanofibre formation is required to achieve reliable transfer of electrospinning-based processes into a relevant industrial environment. The control of electrically-driven jets on large-scale electrospinning plants is one of the most important problems to be solved for real industrial application 
of electrospinning technology. Therefore, the second objective of the work was to introduce a monitoring and control system able to detect electrospinning fault and define the actual state of the process.

Finally, the third objective was to develop an image analysis tool for quality control of the electrospun products (measurements of fibre diameters and pore sizes, identification and quantification of defects), which is still lacking in most of the studies related to electrospinning.

\subsection{State of the Art}

Progresses in the comprehension of the electrospinning process have contributed to the development of functional nanofibres. The variety of polymer-based materials used in electrospinning is steadily growing and now comprises biopolymers and nano-composites. Electrospun nanofibrous materials are physically versatile and multiple chemical functionalities may be introduced using functional polymers [2-6] and/or functional nano-fillers [7-12]. The inclusion of inorganic nanoparticles in electrospinning technique matches with one of the most ambitious challenge in materials science: the creation of multifunctional material by design. Nanoparticles with antibacterial and/or with photo-catalytic/self-cleaning properties can give such new functionalities to the final polymeric product towards the creation of hybrid materials. The extremely high surface area of the electrospun nanofibres can further improve applicability and functionality of the processed materials [5, 6]. For this reason, the huge industrial potential of electrospun nanofibre-based materials claims increased activity. In particular, the proposed development of multifunctional nanofibres by electrospinning, exploitable as filtering media, represents a breakthrough approach for the development of new materials with new functions beyond the state-of-the-art.

The above-mentioned filtering media, which can offer both adsorption and highefficiency filtration properties, can be obtained by electrospinning of functional polymers able to react with pollutant and harmful substances, such as heavy metals, dyes and/or volatile organic compounds (VOCs) like formaldehydes or phenols.

Among the functional polymers, bio-based polymers such as proteins (e.g. keratin) or carbohydrates (e.g. chitosan), offer an interesting solution for the production of nanofibrous filtering media able to adsorb and remove heavy-metal ions, dyes and VOCs. In particular, keratin [13] is characterized by chelating and ionisable groups that can form strong and stable interactions with metal ions, dyes and formaldehyde. Studies on keratin as metal ion adsorbent started in the 1950s, but in most cases they referred to wool fibres, ground wool or feathers. The benefits that could be achieved by the transformation of keratin into nanofibres are not only for filtration (membrane with small pore sizes) but also for adsorption $[2,5,6]$; in fact, due to their high specific surface area, keratin-based nanofibres exhibit enhanced reactivity with respect to wool or feathers. Furthermore, the incorporation of nanoparticles 
into nanofibres is an interesting strategy to confer antimicrobial, self-cleaning and photo-catalytic properties to keratin electrospun nanofibres.

On the other hand, for reaching applications of electrospinning-based processes exploitable at industrial level, some issues have to be solved [14-21]. The most important is the increase in nanofibre output related to the jet stability control. Advances in the knowledge about the complex interconnections between processing conditions/parameters and final products will lead to technical solutions for boosting a cost reliable transfer of electrospinning plants into relevant industrial environment.

The adjustable electrospinning process parameters are mostly flow rate and applied voltage [17]. The flow rate for a single needle setup is typically 0.001-1 $\mathrm{ml} / \mathrm{min}$, depending on the electrospun solution. The low production rate of a single needle electrospinning process may limit the industrial use [18]. Thus to achieve a satisfactory fibre production a high number of needles is required. Although a monitoring strategy by means of the measurement of the voltage drop across a sensing resistor for the single needle apparatus has been already applied, no solution is published for the multi-jet system. In particular, Samatham and Kim [22] measured the current on the grounded side of the system but this solution is not applicable in a multi-jet configuration where monitoring of the current flowing through every single needle, connected to the high voltage source, is needed. A relation between the type of jet, morphology of the deposed fibres and electric current was found. They observed four different types of relations: fluctuating jet associated with beaded nanofibres production, stable jet associated with the production of fibres with uniform diameter, stable jet with spikes corresponding to polymer drops associated with the production of more spherical beads than those formed with fluctuating jet and multiple jet regime associated with the production fibres similar to those obtained from fluctuating jet. The process can be monitored to check the quality of the output by elaborating the current signal in real time. A control loop for a single needle system has been designed using a CCD camera to evaluate the Taylor cone height and comparing it to a target height. When the observed height exceeded the target height, voltage was increased, otherwise it was decreased. A scalable closed loop control system that can maintain a constant source pressure at the capillary was designed and tested by Druesedow et al. [23] to improve the control over the variables of the process that affect the fibre creation. Two sensing technologies based on ultrasound and infrared ray were compared in terms of ability to measure the height of the polymer solution in the container. The air pressure above the solution was detected using a pressure transducer and adjusted by a controllable syringe pump [23].

A constant current electrospinning system has been developed by Munir et al. [24]. The electric current flowing from the collector to ground is measured and kept at a certain value by adjusting the voltage applied to the electrodes. 


\subsection{Problem Statement and Proposed Approach}

Protein processing and sol-gel techniques, and finally production of novel composite nanofibre-based filter media suitable for the treatment of air and water require the development of specific knowledge on material science. Herein the following procedure was adopted for the production of suitable nanofibre-based filter media:

1. Nano-powders and water dispersed nanoparticles (nanosols) were produced separately.

2. The mixing of keratin and inorganic nanoparticles was carried out producing stable solutions.

3. The mixture parameters (colloidal stability, particle size distribution, surface charge) were checked and optimized to achieve electrospinnable formulations.

4. The solutions were characterized and their electrospinnability (i.e. possibility to transform keratin solutions with nanoparticles dispersion into nanofibres by means of electrospinning) was assessed.

5. Novel multifunctional composite nanofibre-based filter media were produced by electrospinning and the resulting nanofibres were tested against bacteria (antibacterial properties) and for the degradation of organic compounds (photocatalytic efficiency).

The details regarding the preparation of the electrospinnable solutions are presented in Sect. 15.4.1.

The production of electrospun nanofibers was realized with a conventional electrospinning apparatus equipped with a novel monitoring system able to measure and control several process parameters. In particular, voltage to the needle and solution flow rate are controlled, and current at the needle, current at the collector, pressure at the needle, environmental temperature and relative humidity are measured. The present monitoring system has been successfully used to detect fault during electrospinning. Moreover, it can speed up the seeking of electrospinning conditions for new polymers, polymer blends or new solvent systems.

Thanks to the measurement of process currents also at the needle, the monitoring system can better characterize process dynamics associated to jet formation and, additionally, separately evaluate each single current in a multi-needle (multi-jet) configuration. Finally, the system is the base of a closed-loop control system for electrospinning.

In order to achieve a reliable control of the electrospinning process, the electric current has been chosen as the main monitoring signal, being correlated, even if not exclusively, to the charges flowing together with the emitted polymeric solution. In order to measure the typical micro-Amperes currents while protecting the electronic circuits from the process high voltages $(10-30 \mathrm{kV})$, an appropriate hardware configuration has to be developed. The main idea is to realize a measuring instrument isolated from ground that will float at the needle high voltage. It is then required to isolate both power supply and data transfer: literature from other technological sectors suggests the adoption of data link based on optical fibres and batteries or 
solar panels (with artificial lighting) or high voltage isolated transformers power supplies. Pearce and James developed a high voltage isolation amplifier with serial data transmission by means of optical fibre [25]. Relevant monitoring systems have been presented by Argirò et al. who developed a current monitoring system for a photomultiplier tube [26] and Sauer et al. [27]. In particular, Sauer designed a nanoammeter floating at $25 \mathrm{kV}$, which can be supplied by a $24 \mathrm{~V}$ battery or by a solar panel and is protected from current spikes and short circuits.

The monitoring and control hardware system developed in this work (Sect. 15.4.2) is composed of two main electronic boxes: a floating voltage instrument box, directly connected to the system at high voltage, which collects process parameters at the needles side and control the flow pump; a grounded instrument box (low voltage) that measures the process current at the collector side and interacts with the high voltage generator, reading the monitoring signals proportional to the supplied voltage and current, and suppling the analogue signal that modulates the generated voltage. The software tool for monitoring and control is presented in Sect. 15.4.3.

In addition, a method to monitor the quality of the produced nanofibrous material has been developed (Sect. 15.4.4).

\subsection{Developed Technologies, Methodologies and Tools}

\subsubsection{Preparation of Electrospinnable Solutions}

For the production of nanocomposite functional nanofibre-based filters, keratin was extracted from wool by sulphitolysis with sodium m-bisulphite. Briefly, wool was cleaned by Soxhlet to remove fatty matter using petroleum ether, washed with distilled water and conditioned at $20{ }^{\circ} \mathrm{C}$ and $65 \%$ relative humidity for $24 \mathrm{~h}$. Then, cleaned and conditioned fibres were cut into snippets of some millimetres and treated with a solution containing urea and sodium m-bisulphite at $\mathrm{pH} 6.5$, for $2 \mathrm{~h}$ at $65{ }^{\circ} \mathrm{C}$. The liquid obtained was filtered with a $5-\mu \mathrm{m}$ pore-sized filter. Then, dialysis was carried out for 3 days against distilled water using cellulose tubes with $12-14 \mathrm{kDa}$ of molecular cut-off. After dialysis, the resulting liquid was freeze-dried obtaining pure keratin powder. Titanium dioxide $\left(\mathrm{TiO}_{2}\right)$ sol (NAMA41, $6 \mathrm{wt} \%$ at $\left.\mathrm{pH} 1.5\right)$ and silver (Ag) sol (NAMA39, $4 \mathrm{wt} \%$ at $\mathrm{pH} 4$ ) supplied by Colorobbia Italia (Italy), were used to add colloids in the electrospinning feed. In order to evaluate the stability of nanosol in poly(ethylene oxide) (PEO, Mwa $400 \mathrm{kDa}$ by Sigma Aldrich, Italy), some dispersion tests without keratin were performed. Aiming to assess nanoparticles stability on the PEO solutions, Dynamic Light Scattering (DLS) was used by measuring the hydrodynamic diameter of particles and assessing the particle size distribution. Measurements were carried out by Zetasizer Nanoseries apparatus (Malvern, UK) while working at the fixed angle of $173^{\circ}$. Samples were properly diluted in water and poured in a polystyrene cuvette. Hydrodynamic diameter measures the coordination sphere and species adsorbed on the particle surface. 


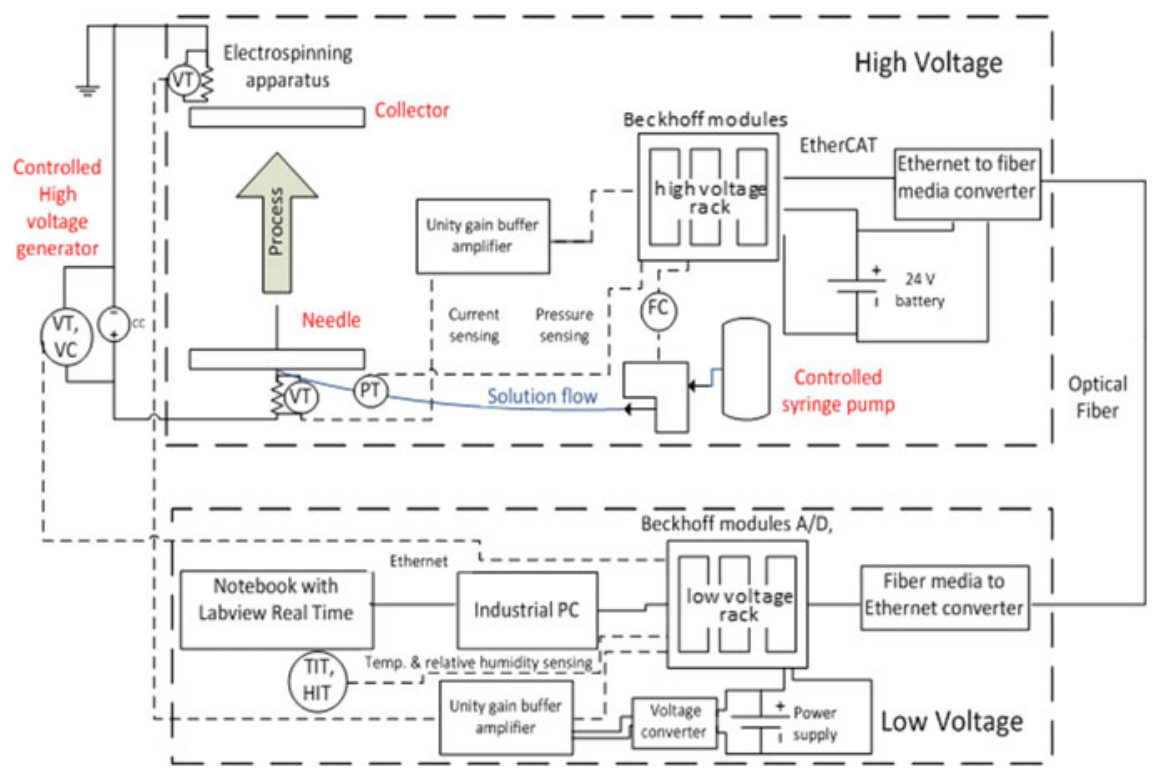

Fig. 15.2 Layout of the monitoring and control hardware system

Suspensions containing inorganic nanoparticles, PEO and keratin were prepared as follows for electrospinning. Freeze-dried keratin powder was added to de-ionized water until a concentration of $7 \mathrm{wt} \%$ and stirred at room temperature for $18 \mathrm{~h}$. Similarly, PEO was dissolved in distilled water until a concentration of $7 \mathrm{wt} \%$. After $6 \mathrm{~h}$ under stirring, nanosols were introduced into the PEO solution and kept under stirring for $18 \mathrm{~h}$. The resulting PEO solutions with nanosols and keratin were mixed at 30:70 weight ratio, and stirred for at least $2 \mathrm{~h}$ before electrospinning. The nanosols were added to the PEO solutions reaching a nanoparticle concentration of $3 \mathrm{wt} \%$ with respect to the final keratin content. When silver nanosol was used, an anionic surfactant, i.e. sodium dodecyl sulfate, was added at a concentration of $1 \mathrm{wt} \%$ on the keratin content in order to avoid protein precipitation.

\subsubsection{Monitoring and Control Hardware for Electrospinning}

The monitoring and control hardware system for electrospinning is illustrated in Fig. 15.2 and includes:

1. High voltage instrument box.

2. EtherCAT connection via optical fibre.

3. Low voltage instrument box. 
4. Given the low input impedance of the industrial A/D modules and the high value of the current metering resistors (i.e. $12 \mathrm{M} \Omega$ ), appropriate high input impedance Instrumentation Amplifiers have been installed (4 channels).

5. An industrial PC with Real Time operating system by National Instruments. The unit acts as master on the EtherCAT bus and via a standard Ethernet line is connected to user-interface PC.

6. Standard Windows PC, with non-real time monitoring SW and User Interface, developed in Labview, National Instruments.

7. Canon EOS 60D camera, to record HD video of the needle zone.

The High voltage instrument box is referred to the needle high voltage (10-25 kV) and isolated from ground. It measures, for maximum three needles, their process currents and syringe supply pressures. Additionally, it controls the pump that operates on three syringes, imposing the same feeding speed. The High voltage instrument box contains:

- Beckhoff modules; i.e. EK1541 coupler with optical fibre connection; two EL3104: 4-channels analogue input $( \pm 10 \mathrm{~V}, 16$ bits); EL7031 stepper motor controller, connected to a Beckhoff AS1030 stepper motor.

- $24 \mathrm{~V}$ battery.

The Low voltage instrument box is referred to ground and it measures the process current at the collector side and interacts with the high voltage generator (Spellman SL 50), reading the monitoring signals proportional to the generated voltage and current, and supplying the analogue signal that modulates the generated voltage. The Low voltage instrument box contains:

- Beckhoff modules, i.e. two EL3602 2-channel analogue input $( \pm 10, \pm 5, \pm 2.5 \mathrm{~V}$, 24 bits), EL4132 2-channel analogue output terminals ( $\pm 10 \mathrm{~V}, 16$ bits).

- Ambient temperature and relative humidity sensor.

\subsubsection{Software Tool for Monitoring and Control}

The software tool for monitoring and control has been developed in LabVIEW and consists of two parts, one running on the non real-time PC, with Microsoft Windows, and one running on the real-time industrial PC. The former is organized in three parts:

- Graphic user interface

- Messages handling

- Target-Host communication.

It has a case-event structure (State Machine), which is handled by a standard Labview SW architecture, called Queued Message Handler (QMH). QMH is the combination of a producer event handler that pushes user messages onto a queue, and a consumer with an embedded state machine that can push its own messages onto the queue. 
The pump can be operated manually by the operator through the pump control panel, setting the flow rate and selecting the syringe diameter, or automatically by the control SW, currently under development.

The software program running on the industrial PC consists of seven parts:

- Acquisition Module

- Elaboration Module

- Logging Module

- Pump Module

- Message Handling Loop

- Target-Host Connection.

Commands are sent from the Host to the target via the Target-Host connection and handled by the Message Handling Loop. All parts are constituted by a case structure running in a while loop. Communication between parts is handled by the QMH.

Acquisition is handled by the Acquisition Module (12 channels, 8 from the high voltage section and 4 from the low voltage one) which also sends data to the Elaboration Module, to the Logging Module and to the Pump Module. In the Elaboration Module, several scalar indexes are calculated to extract information about process. The average value, variance, number of peaks, energy, skewness, kurtosis, mean peak frequency and actual peak frequency are computed on a moving window and are shown to the operator.

The Logging Module receives data from the Acquisition and Elaboration Modules and saves it in two separate text files.

The Pump Module handles the pump speed in order to obtain the desired flow rate set by the operator. In fact the actual flow rate at the needle is equal to the pump flow rate only at steady state, because, during transients, part of the pump flow compensates for piping compliance, which is significant, compared to the small flow rate required by the process. To reduce the transient time, a pump controller has been developed based on the pressure signal measured near the needle by the installed pressure sensor. The relationship between the actual needle flow rate and the measured pressure, which depends on solution viscosity and needle geometry, was experimentally identified, performing tests with different constant pump flow rates, waiting until the steady state was reached.

\subsubsection{Image Analysis Tool for Quality Control of the Electrospun Products}

Several methods have been proposed in the literature to monitor the quality of produced nanofibrous materials. These methods can be classified according to the control strategy, i.e. (1) continuous control of the production parameters or (2) spot checks of the produced materials. Monitoring production parameters is simpler, but the quality of the produced materials can be affected by several other factors; thus, an inspection of the produced material is more suited. Two options are possible: to directly analyse 
the structure of the nanofibrous material by SEM (scanning electron microscope) imaging, or to assess some functional properties. As the latter option does not allow generating alerts in a short time, the most effective approach entails to attain SEM images of few material samples and observe the morphology. Outcomes from the spot analysis are then used to create a statistical inference on the entire production process [28]. However, the existing solutions to analyse SEM images of nanofibrous materials are only meant to measure fibres diameter and orientation [29-31], but it is not possible to detect the typical defects that are present in nanofibrous materials. Thus, a defect/anomaly detection solution based on a dictionary yielding sparse representations has been developed. In particular, the approach proposed in [32] to represent normal data in terms of a dictionary learned during an early training stage has been used. Then, test images are analysed in a patch-wise way, computing features to assess the conformation of each patch with the morphologies characterising the normal ones. Anomalies are recognised as outliers in the feature distribution, and the outcome of the analysis is the percentage of pixels associated with a defect [28].

The procedure previously described has been validated considering 45 SEM images (dimension $1024 \times 696$ pixels) acquired with the Field Emission Scanning Electron Microscope (Carl Zeiss Sigma NTS, GmbH Öberkochen, Germany). Indeed, specimens of $4 \times 4 \mathrm{~cm}$ were collected on the produced material and placed on a metallic support. A thin gold coating of about $5 \mathrm{~nm}$ was applied on the sample surface to guarantee satisfactory electrical conduction by sputter-coating. All images were acquired with magnification $8000 \mathrm{x}$, extra high tension $5 \mathrm{kV}$, working distance $7 \mathrm{~mm}$, brightness $45 \%$, and contrast 52\%. Our dataset contains 45 SEM images: 5 images are anomaly free, whereas 40 images contain anomalies of different size [28].

To assess the performance of the proposed method, we have considered the ratio between the False Positive Rate (FPR), i.e., the percentage of pixels erroneously identified as anomalous, and the True Positive Rate (TPR), i.e., the percentage of the number of pixels properly identified as anomalous. Performances are globally evaluated through the Receiver Operating Characteristic (ROC) curve acquired by plotting the TPR against the FPR for different values of a threshold that can be tuned by the operator. The Area Under the ROC Curve (AUC) is the common performance indicator usually adopted in the literature, which is at most equal to 1 in the case of the perfect detector (i.e., when TPR $=100 \%$ and $\mathrm{FPR}=0 \%$ ) [28].

We have compared our algorithm against 5 state-of-the-art anomaly detection systems, tested under the same setting. Our approach outperforms the others, with an AUC of 0.926 against AUCs ranging between 0.619 and 0.926 for the other solutions. Details about the proposed solutions and the validation outcomes can be found in [28], while the code and some test images are available for download. ${ }^{1}$

\footnotetext{
${ }^{1}$ web.mi.imati.cnr.it/ettore/NanoTWICE.
} 


\subsection{Testing and Validation of Results}

The system has been tested in process conditions using the materials and setup presented in Sect. 15.5.1. A set of experiment was designed to test the monitoring system (Sect. 15.5.2). Finally, a new set of conditions has been defined to validate the electrospinning prototype plant equipped with the monitoring and control system (Sect. 15.5.3).

\subsubsection{Materials and Basic Electrospinning Setup Description}

Poly(ethylene oxide) (PEO, average Mv $400 \mathrm{kDa}$, from Sigma Aldrich) was dissolved in distilled water at room temperature for about $12 \mathrm{~h}$ under magnetic stirring. All experiments were carried out at $7 \%$ wt. of concentration. PEO has been selected because of its well-known electrospinnability and huge literature available.

Suspensions containing inorganic nanoparticles, PEO and keratin were prepared as described in Sect. 15.4.1.

The solutions were electrospun using an electrospinning apparatus that comprises a plastic syringe containing about $5 \mathrm{~mL}$ of solution, and a high-precision syringe pump developed ad hoc by the authors. The pump is feed but the battery of the instrumentation box floating at high voltage to avoid current leakage toward ground (which can damage pump isolation and generate noise in the measured currents). Additionally, the pump speed is controlled by the supervising computer. A stainless steel needle (gauge 27) with an internal diameter of $0.2 \mathrm{~mm}$ was connected to the syringe and to a SL50 high-voltage generator (Spellman, UK). A stainless steel plate $(20 \mathrm{~cm}$ by $20 \mathrm{~cm}$ ) was positioned in front of the needle as collector, which was electrically grounded at $10-20 \mathrm{~cm}$ distance.

\subsubsection{Experiments and Analysis of the Process}

The list of experiments performed on the monitoring system is reported in Table 15.1. Values of the parameters were defined by Design of Experiments using the experiments of a previous work [33], to explore process conditions near the optimal values.

During the experiments, seven different states of the process were identified. States are described in the following as observed during the experimental campaign.

State 1 No solution occurs when the voltage power supply is turned on and the process voltage is applied to the tip, but there is no polymer solution available to support the formation of a jet. Even if there is no fibre formation, the current measured is comparable to the normal electrospinning process, because of significant air ionization near the needle. In that case, the pressure at the tip, as there is no polymer 
Table 15.1 List of the parameters defined by design of experiments

\begin{tabular}{|c|c|c|c|}
\hline $\begin{array}{l}\text { Experiment } \\
\text { no. }\end{array}$ & Voltage (kV) & $\begin{array}{l}\text { Flow rate } \\
(\mathrm{ml} / \mathrm{min})\end{array}$ & Distance $(\mathrm{cm})$ \\
\hline 1 & 23 & 0.02 & 22 \\
\hline 2 & 20 & 0.01 & 22 \\
\hline 3 & 20 & 0.02 & 22 \\
\hline 5 & 28 & 0.01 & 22 \\
\hline 6 & 28 & 0.02 & 22 \\
\hline 8 & 23 & 0.01 & 22 \\
\hline 10 & 20 & 0.01 & 22 \\
\hline 11 & 23 & 0.02 & 22 \\
\hline 13 & 18 & 0.005 & 22 \\
\hline 14 & 28 & 0.02 & 22 \\
\hline 16 & 20 & 0.02 & 22 \\
\hline 18 & 23 & 0.01 & 22 \\
\hline 20 & 18 & 0.005 & 22 \\
\hline 21 & 23 & 0.02 & 22 \\
\hline 22 & 28 & 0.01 & 22 \\
\hline 24 & 26 & 0.03 & 28 \\
\hline 25 & 26 & 0.03 & 28 \\
\hline 26 & 24 & 0.025 & 28 \\
\hline 27 & 28 & 0.025 & 28 \\
\hline 28 & 24 & 0.025 & 28 \\
\hline 29 & 28 & 0.025 & 28 \\
\hline 30 & 24 & 0.025 & 25 \\
\hline 31 & 28 & 0.025 & 25 \\
\hline 32 & 24 & 0.025 & 25 \\
\hline 33 & 28 & 0.025 & 25 \\
\hline
\end{tabular}

solution in the system, is equal or near to the ambient pressure. A light indicator on the front panel of the monitoring software turns on, suggesting the operator to check the solution supply.

State 2 Drop formation occurs when the solution flow rate to the needle is too high, compared to the flow rate of the emitted jet, the drop at the needle tip increases in size. If no countermeasures are implemented, after a variable time depending on process parameters, the spinning stops. Therefore, State 2 can lead to State 6 No electrospinning, see below. Moreover, during State 2, it is also possible that part of the solution fed to the needle is projected from the tip as small droplets that reach the collector, forming defects, similar to a film on the nanofibre mat, as show in Fig. 15.3a. At the needle, instable jets and droplets are produced in a short sequence, as reported in Fig. 15.3b. When that happens, peaks in the current signal occur: the monitoring system identifies those peaks, as shown in Fig. 15.3c, and calculates their total number and repetition frequency. 
Fig. 15.3 a SEM picture of nanofibers with film defects. b Pictures of an electrospinning tip at State 2 from the video camera. c Current signals measured during State 2: drop ejection
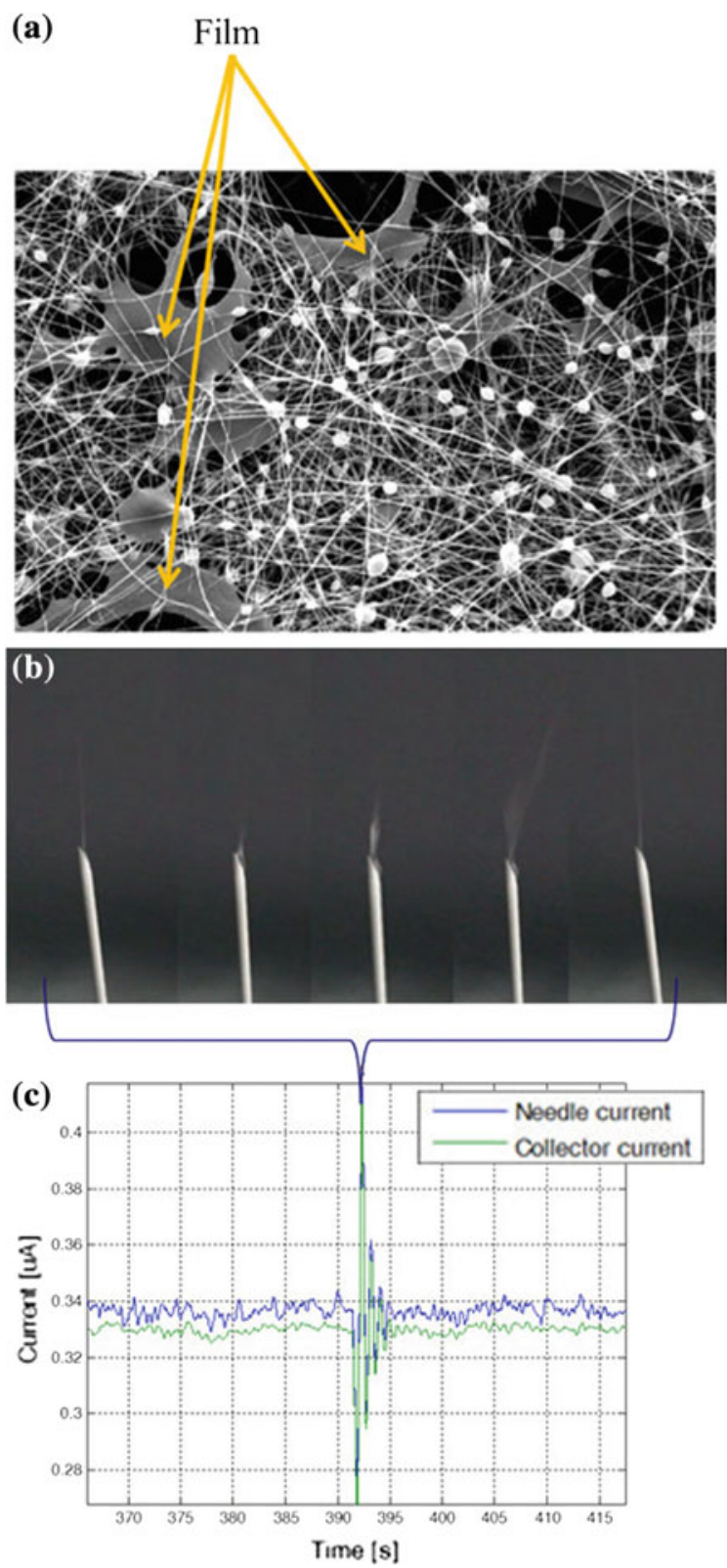

The control strategy proposed in the case of High flow rate is to decrease the flow rate or to increase the voltage, or a combination thereof. Particular attention must be given when the voltage is increased because the jet can reach the collector not completely dry, because of the reduced time of flight. 
Fig. 15.4 SEM picture of nanofibers with beads (a). Pictures of an electrospinning tip at State 3 from the video camera (b). Current signals measured during State 3 (c)
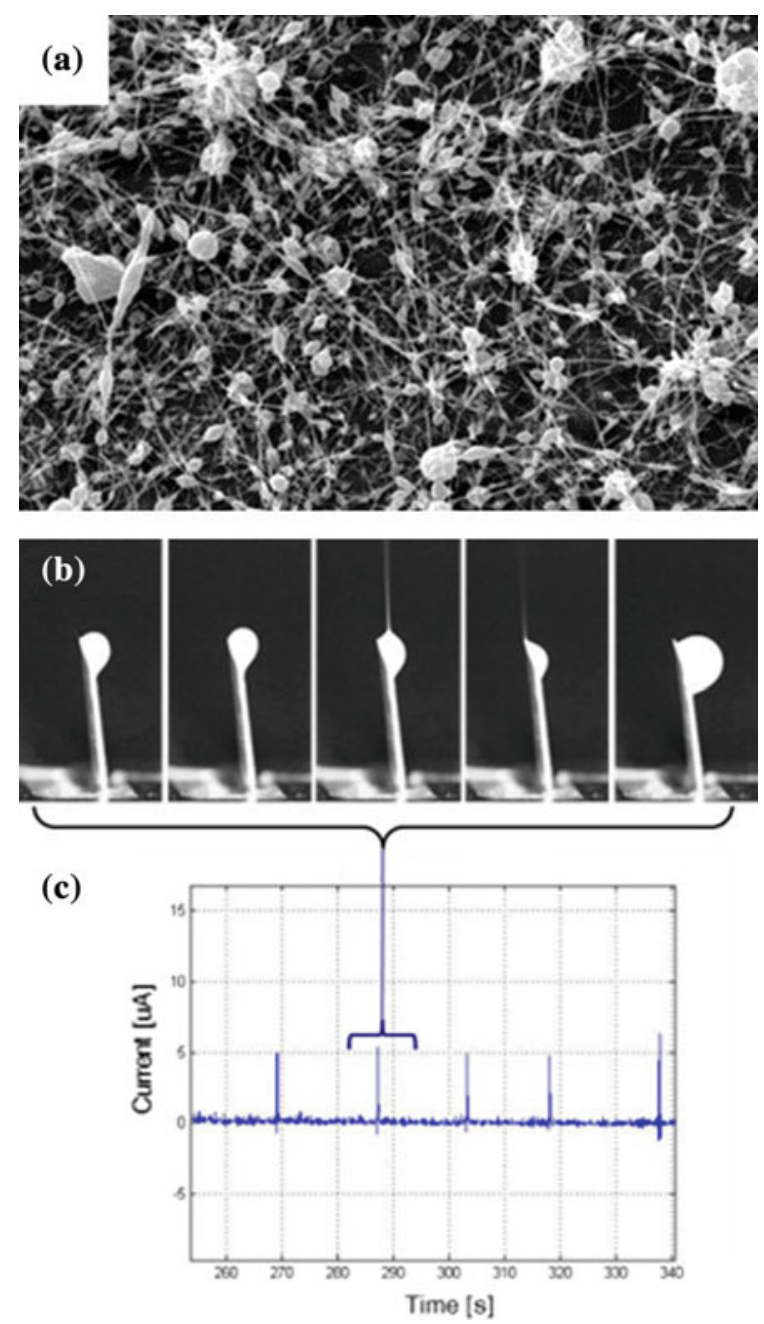

State 3 Intermittent flow occurs when the needle is fed with a flow rate lower than the one requested by a stable jet, the throughput of the electrospinning plant is limited and an intermittent jet can be observed. Intermittent jet is associated with a large production of beads, as shown in Fig. 15.4a. When an intermittent jet occurs (Fig. 15.4b), an intermittent current signal is measured, as reported in Fig. 15.4c. When the needle is not producing a jet, the current measured is negligible because the drop on the needle tip acts like an electric shield (compared to State 1).

State 4 is Stable jet and it is related to a steady electrospinning and good nanofibre production, as shown in Fig. 15.5a. A stable jet (Fig. 15.5b) is associated with a stable electric current, as Fig. 15.5c shows. In this case, nanofibres have uniform diameter with no beads and defects, according to Samatham et al. [22]. 
Fig. 15.5 SEM picture of nanofibers without defects (a). Picture of an electrospinning tip at State 4 from the video camera $(\mathbf{b})$. Current signals measured during State 4 (c)

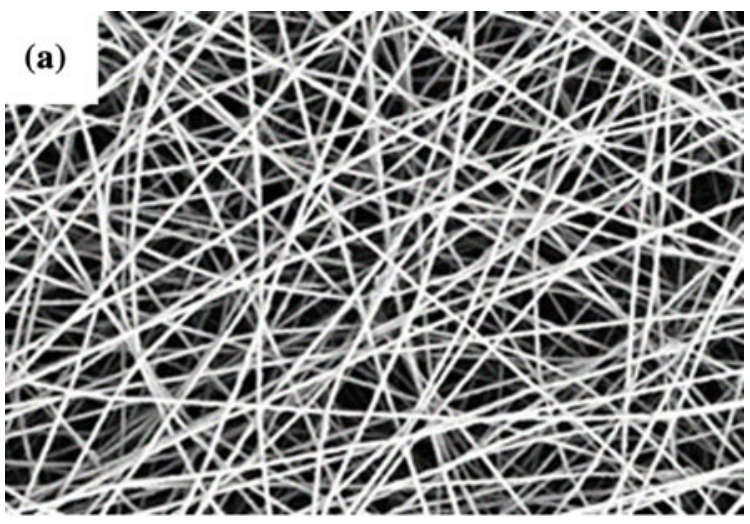

(b)

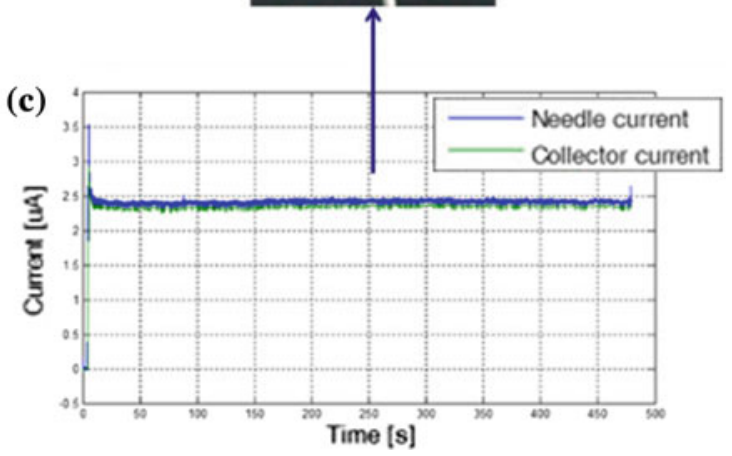

In some experimental conditions (high voltage and high flow rate), a multitude of jets were observed emerging from the needle tip (Fig. 15.6a). This is the State 5 Multiple jets. The electric current recorded during this phenomenon decreased. The finding disagrees with previous works [22]. The major cause of such difference is likely due to the geometry of the electrospinning equipment because the size of the collector could be not enough to intercept all the emitted jets.

State 6 is No electrospinning and it happens when the solution is fed to the needle at regular pressure, but no jet is ejected from the tip. Thus, the solution accumulates at the tip forming a large drop, as show in Fig. 15.6b. In this state the current signal is lower than the normal electrospinning current. 

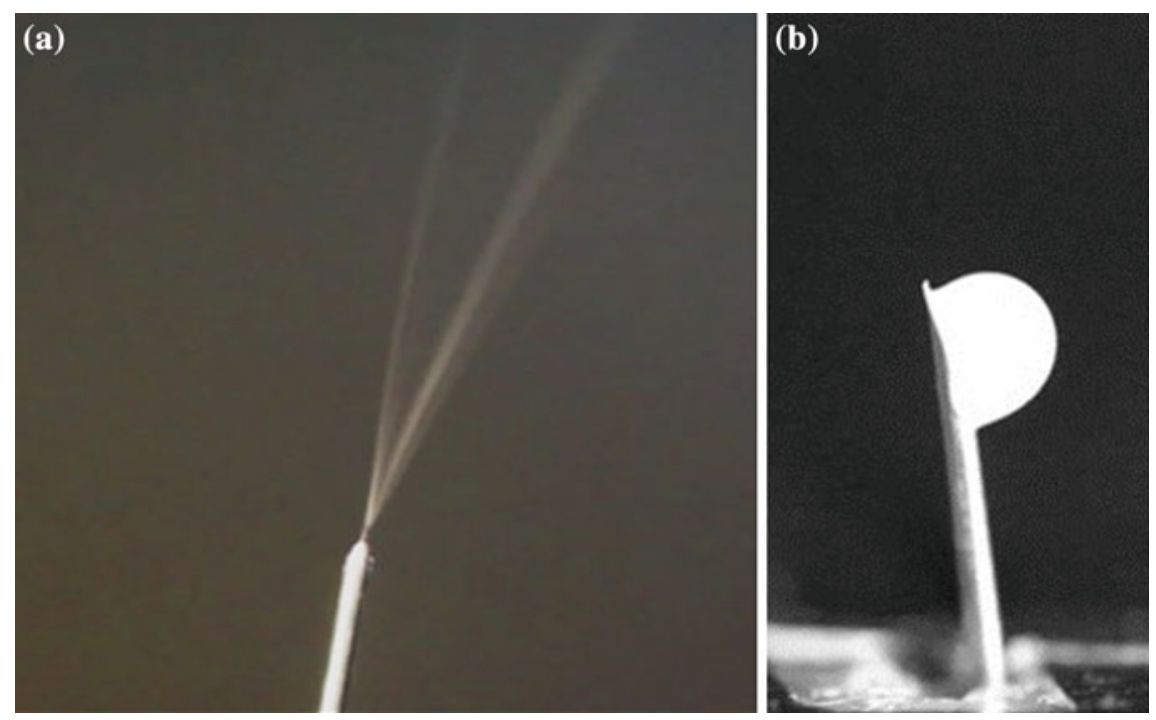

Fig. 15.6 Pictures of an electrospinning tip at State 5 (a) and State 6 (b) from the video camera

State 6 can be a worsening of the State 2. Finally, the State 7 is related to the clogging of the needle due to solidification of the solution. No electrospinning occurs, while pressure at the needle supply increases.

A new set of four experiments was carried out in order to validate the previous observations of the monitoring system employing operative conditions that lead to stable jet. Distance and flow rate were fixed at $22 \mathrm{~cm}$ and $0.03 \mathrm{ml} / \mathrm{min}$ respectively. Voltage was set at 22, 25, 28 and $30 \mathrm{kV}$ in experiments labelled $A, B, C$ and $D$, respectively. At $A-D$ conditions, the process is stable and good nanofibres were produced. Moreover, the size of the drop at the needle tip decreases as the voltage increases.

\subsubsection{Nanofibre-Based Filter Production and Testing}

For the production of nanocomposite functional nanofibre-based filters, processing conditions were investigated (flow-rate from 0.001 to $0.05 \mathrm{ml} / \mathrm{min}$, voltage from 20 to $35 \mathrm{kV}$ and tip-to-collector distance from 15 to $30 \mathrm{~cm}$ ). In electrospinning, temperature and relative humidity can affect process stability and nanofibre morphology; therefore, environmental conditions of the electrospinning room were recorded using an Escort RH iLog datalogger and adjusted accordingly. The temperature was maintained between 25 and $30^{\circ} \mathrm{C}$, and the relative humidity was maintained in the range 40-50\%. Based on the previous test results, optimal experimental electrospinning 


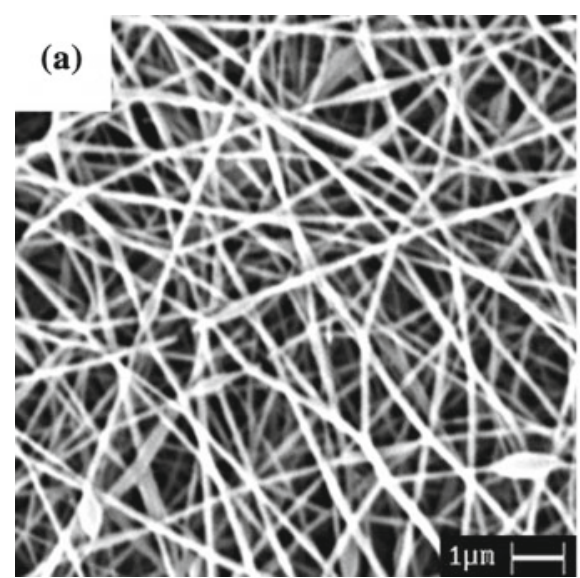

Fig. 15.7 SEM pictures of keratin nanofiber titania nanoparticles $(\mathbf{b})$

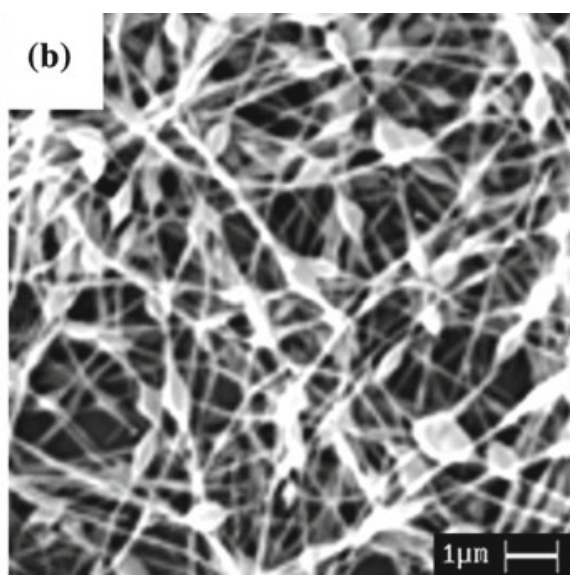

(a) and

parameters were: applied voltage $25 \mathrm{kV}$, flow rate $0.01 \mathrm{ml} / \mathrm{min}$ and tip-to-collector distance $22 \mathrm{~cm}$, in good agreement with the model [33].

Nanofibres were characterized by infrared spectroscopy and SEM before and after the heat treatments and after water filtration. Infrared spectra were carried out by means of a Thermo Nicolet Nexus spectrometer by Attenuated Total Reflection (ATR) technique using a Smart Endurance accessory (diamond crystal $\mathrm{ZnSe}$ focusing element). The spectra were collected in the range from 4000 to $550 \mathrm{~cm}^{-1}$ with 100 scansions at $4 \mathrm{~cm}^{-1}$ of band resolution. Omnic 6.2 software tool was employed to record the spectra and to perform ATR baseline correction. The presence of keratin was assessed and morphology of the nanofibres does not change due to treatments, as Fig. 15.7 shows.

Antibacterial tests against Escherichia coli (ATCC 11229) on electrospun nanofibres with silver and titanium dioxide were based on the AATCC 100 Test Method. The procedure was the following:

(a) The electrospun keratin/PEO samples with titanium dioxide and the corresponding reference samples without nanoparticles were contacted with $1 \mathrm{ml}$ of bacteria inoculum after activation by UV irradiation. The bacteria inoculum contains $1.5-3 \times 10^{5} \mathrm{CFU} / \mathrm{ml}$. The samples were exposed to UV light at $20^{\circ} \mathrm{C}$ and $65 \%$ $\mathrm{RH}$ for $1 \mathrm{~h}$. After this time, $100 \mathrm{ml}$ of sterile buffer were added on each sample. The buffer was then diluted ten times and pleated on Petri dishes with agar. The Petri dishes were incubated at $37^{\circ} \mathrm{C}$ for at least $24 \mathrm{~h}$, and then the bacteria colonies were counted.

(b) The samples with silver nanoparticles and on the corresponding reference sample without nanoparticles were contacted with $1 \mathrm{ml}$ of bacteria inoculum containing 1.5-3 $\times 10^{5} \mathrm{CFU} / \mathrm{ml}$. Then the samples were incubated for $1 \mathrm{~h}$ at $37^{\circ} \mathrm{C}$. After this time, $100 \mathrm{ml}$ of sterile buffer were added to each sample. The buffer 
was then dilute ten times and pleated on Petri dishes with agar. The Petri dishes were incubated at $37^{\circ} \mathrm{C}$ for at least $24 \mathrm{~h}$, and then the bacteria colonies were counted.

Bacterial reduction is calculated according to Eq. (15.1), where $A$ represents the number of colonies of the samples with nanoparticles, whereas $B$ represents the number of colonies of the samples without nanoparticles (references).

$$
\% \text { bacterial reduction }=\frac{B-A}{B} \cdot 100
$$

Photocatalytic tests were performed measuring the degradation of the Rhodamine $\mathrm{B}$ dye $(\mathrm{RhB})$ in water as a model organic compound. The photocatalytic degradation of $\mathrm{RhB}$ by UV-irradiation was carried out at room temperature using samples of electrospun nanofibres containing $3 \% \mathrm{wt}$. of $\mathrm{TiO}_{2}$. Before irradiation, the system was kept in the dark for $1 \mathrm{~h}$ to stabilize an adsorption-desorption equilibrium between nanofibres and $\mathrm{RhB}$ dye. The solution containing the electrospun nanofibre sample was irradiated with intensity of $9 \mathrm{~W} / \mathrm{cm}^{2}$ (Osram ULTRA-Vitalux $300 \mathrm{~W}$ lamp) keeping the solution stirred. In order to ensure a steady spectral emission the UV lamp was switched on $30 \mathrm{~min}$ before the photocatalytic test. The degradation reaction was regularly checked by taking $3 \mathrm{~mL}$ of solution for the measure of the absorbance at the wavelength of maximum absorbance of $\mathrm{RhB}$ (at $554 \mathrm{~nm}$ ) with a single beam spectrophotometer (UV/Vis Spectrophotometer S-22, Boeco, Germany). According to the Lambert-Beer law, the absorbance is proportional to the RhB concentration, this way the photocatalytic efficiency was calculated as conversion (\%) over the time of irradiation.

The so-obtained conversion refers to the ratio between the amount of reagent consumed and the starting amount of reagent following Eq. (15.2), where $A_{0}$ is the initial absorbance and $A_{\mathrm{t}}$ the absorbance after a certain irradiation time $t$.

$$
\text { Conversion }(\%)=\frac{A_{0}-A_{t}}{A_{0}} \cdot 100
$$

A sample without the addition of $\mathrm{TiO}_{2}$ was considered as reference. Antibacterial tests were carried out using AATCC 100 Test Method on nanofibres with silver and titanium dioxide and compared to the nanofibres without nanoparticles. The results were $97 \%$ of bacteria reduction for nanofibres with titania nanoparticles and $95 \%$ of bacteria reduction for nanofibres with silver nanoparticles. Nanofibres showed excellent antibacterial properties as a sign that the nanoparticles maintain their functions even if inserted in the keratin matrix of the electrospun nanofibres.

Photocatalytic tests based on model reaction of $\mathrm{RhB}$ dye degradation showed promising photocatalytic properties for the hybrid electrospun nanofibres. The results in terms of conversion values were $53 \%$ for keratin nanofibres with titania nanoparticles, $14 \%$ for keratin nanofibres without nanoparticles and $12 \%$ for control solution (without electrospun materials). 
An approach to optimize the process parameters in order to fit some requirements on the produced material while reducing its defectiveness has been developed. It is based on a statistical Design of Experiments (DOE) framework with three response variables related to both the defects and the structural characteristics of the fibres i.e., the defect ratio, the fibre diameter, and the size of pores. DOE can help to study the correlation between fibre diameter and process factors [34], but the analyses were limited to factors that exhibit an influence only on one process output and built up regression models to inspect only these specific relationships.

A more flexible tool has been developed. In particular, exploiting the Response Surface Methodology (RSM) idea, a 23 central composite design based on 20 runs is adopted [35], thus obtaining a regression model for each output variable in function of both controlled process parameters (polymer concentration, polymer feed rate, and applied voltage) and uncontrolled environmental factors, (room temperature and relative humidity), which are known to affect the fibre mat structure. Output data are obtained from SEM images of electrospun nanofibrous materials, as described above, while input data are either imposed or measured during the process.

The regression models are combined into a constrained optimization problem: response surfaces of pore and fibre diameter are used to detect an acceptable region, where the minimum of the defect surface is searched. The use of process parameters obtained in this manner produce a fibre mat with the desired morphology (porosity and diameter are in the desired range of values) and guarantee that presence of defects is as less as possible.

This approach has been applied to polyethylene oxide (PEO) on the prototype electrospinning apparatus. In particular, three examples of constrained optimization were analysed in that work to show outcomes of the proposed optimization approach. Large admissible intervals for fibre diameter and the size of pores were defined in the first test to explore the optimal surface on a wide domain, while narrower intervals were considered in the following tests, to represent realistic and specific production requests. Optimization outcomes are in line with the experience and allow to properly define the process parameters to set based on the desired material properties. Detailed results of the tests and some insights on the estimated surfaces can be found in [33].

\subsection{Conclusions and Future Research}

Functional inorganic nanoparticles of silver and titania were embedded into a protein matrix of keratin in order to produce hybrid organic/inorganic nanofibres by electrospinning. The use of colloids in solutions suitable for electrospinning allows the production of multi-functional nanofibres. Keratin (a protein extracted from wool) has been used as polymer matrix because of its properties in adsorption of dye, VOCs and metals, titania was selected for its excellent photocatalytic and antibacterial capacity, and silver for antibacterial and antifouling properties.

This work demonstrates the possibility to use in electrospinning water, instead of harmful chemicals, as solvent for the production of keratin-based nanocomposite 
nanofibres with multifunctional properties suitable for both air and water treatments and depuration. On the other hand, there are clear practical advantages in using aqueous colloids instead of nano-powders (e.g. attain good dispersion of nanoparticles in the polymer nanofibres, overcome safety issues). The main practical benefit of embedding colloids in electrospun nanofibres is that both unprocessed and final materials can be handled more easily than nanoparticle powders.

Finally, functionalities of the nanoparticles embedded in the nanofibres in degrading organic compounds and/or in killing microorganisms were assessed. The results demonstrate that electrospun keratin-based nanofibres with a high surface area guarantee preserving nanoparticle properties.

As for the analysis of the images and the evaluation of the optimal working conditions, the developed methodology for analysing the defects proved to be effective and to accurately map pixels of a SEM image associated to a defect in few minutes. From that, it is possible to obtain indicators mediated over the acquired images to be used as final quality control of the production.

The developed monitoring system can monitor the needle current by an innovative instrument floating at high voltage. Tests have shown that current at the needle side carries more information about the jet producing project, compared to the current at the collector side, usually used for process monitoring in the literature. This allows an early diagnosis of solution accumulation at the needle tip, useful to avoid drop emission and intermittent and/or low quality electrospinning. The developed monitoring system integrates needle and collector current measurements with supply pressure measurement at the needle. The system has been designed to monitor in parallel up to three emitting needles, to support multi-jets configurations.

Acknowledgements This work has been funded by the Italian Ministry of Education, Universities and Research (MIUR) under the Flagship Project "Factories of the Future-Italy" (Progetto Bandiera "La Fabbrica del Futuro") [36], Sottoprogetto 1, research projects "composite Nanofibres for Treatment of air and Water by an Industrial Conception of Electrospinning" (NanoTWICE) and "Automated electrospinning plant for industrial manufacturing of functional composite nanofibres" (AUTOSPIN).

\section{References}

1. Varesano A, Carletto RA, Mazzuchetti G (2009) Experimental investigations on the multi-jet electrospinning process. J Mater Proc Technol 209(11):5178-5185

2. Aluigi A, Vineis C, Tonin C, Tonetti C, Varesano A, Mazzuchetti G (2009) Wool keratin-based nanofibres for active filtration of air and water. J Biobased Mater Bioen 3:311-319

3. Baek DH, Ki CS, Um IC, Park YH (2007) Metal ion adsorbability of electrospun wool keratose/silk fibroin blend nanofiber mats. Fibers Polym 8:271-277

4. Aluigi A, Tonetti C, Vineis C, Varesano A, Tonin C, Casasola R (2012) Study on the adsorption of chromium (VI) by hydrolyzed keratin/polyamide 6 blend nanofibres. J Nanosci Nanotechnol 12:7250-7259

5. Aluigi A, Tonetti C, Vineis C, Tonin C, Mazzuchetti G (2011) Adsorption of copper(II) ions by keratin/PA6 blend nanofibres. Eur Polym J 47:1756-1764 
6. Aluigi A, Rombaldoni F, Tonetti C, Jannoke L (2014) Study of Methylene Blue adsorption on keratin nanofibrous membranes. J Hazard Mater 268:156-165

7. Linsebigler AL, Lu G, Yates JT (1995) Review-photocatalysis on $\mathrm{TiO}_{2}$ surfaces: principles, mechanisms, and selected results. Chem Rev 95:735-758

8. Hoffmann MR, Martin ST, Choi W, Bahnemann DW (1995) Environmental applications of semiconductor photocatalysis. Chem Rev 95:69-96

9. Costa A, Ortelli S, Blosi M, Albonetti S, Vaccari A, Dondi M (2013) $\mathrm{TiO}_{2}$ based photocatalytic coatings: from nanostructure to functional properties. Chem Eng J 225:880-886

10. Fujishima A, Honda K (1972) Electrochemical photolysis of water at a semiconductor electrode. Nature 238:37-38

11. Ortelli S, Blosi M, Albonetti S, Vaccari A, Dondi M, Costa AL (2013) $\mathrm{TiO}_{2}$ based nanophotocatalysis immobilized on cellulose substrates. J Photochem Photobiol A Chem 276:58-64

12. Rai M, Yadav A, Gade A (2009) Silver nanoparticles as a new generation of antimicrobials. Biotechnol Adv 27:76-83

13. Varesano A, Vineis C, Tonetti C, Sánchez Ramírez DO, Mazzuchetti G (2014) Chemical and physical modifications of electrospun keratin nanofibers induced by heating treatments. J Appl Polym Sci 131:40532

14. Tomaszewski W, Szadkowski M (2005) Investigation of electrospinning with the use of a multi-jet electrospinning head. Fibres Text East Eur 13(4):22-26

15. Kim GH, Cho YS, Kim WD (2006) Stability analysis for multi jets electrospinning process modified with a cylindrical electrode. Eur Polym J 42(9):2031-2038

16. Kumar A, Wei M, Barry C, Chen J, Mead J (2010) Controlling fiber repulsion in multijet electrospinning for higher throughput. Macromol Mater Eng 295(8):701-708

17. Varesano A, Rombaldoni F, Mazzuchetti G, Tonin C, Comotto R (2010) Multi-jet nozzle electrospinning on textile substrates: observations on process and nanofibre mat deposition. Polym Int 59(12):1606-1615

18. Yang Y, Jia Z, Li Q, Hou L, Liu J, Wang, L et al (2010) A shield ring enhanced equilateral hexagon distributed multi-needle electrospinning spinneret. IEEE Trans Dielectr Electr Insul 17(5):1592-1601

19. Angammana CJ, Jayaram SH (2011) The effects of electric field on the multijet electrospinning process and fiber morphology. IEEE Trans Ind Appl 47(2):1028-1035

20. Zheng Y, Zhuang C, Gong RH, Zeng Y (2014) Electric field design for multijet electropsinning with uniform electric field. Ind Eng Chem Res 53(38):14876-14884

21. Zheng Y, Gong RH, Zeng Y (2015) Multijet motion and deviation in electrospinning. RSC Adv 5(60):48533-48540

22. Samatham R, Kim KJ (2006) Electric current as a control variable in the electrospinning process. Polym Eng Sci 46(7):954-959

23. Druesedow CJ, Batur C, Cakmak M, Yalcin B (2010) Pressure control system for electrospinning process. Polym Eng Sci 50(4):800-810

24. Munir MM, Iskandar F, Khairurrijal OK (2008) A constant-current electrospinning system for production of high quality nanofibers. Rev Sci Instrum 79(9):093904

25. Pearce JW (1978) Optically coupled high voltage isolation amplifier. Rev Sci Instrum 49(11): 1562

26. Argirò S, Camin DV, Destro M, Guérard CK (1999) Monitoring DC anode current of a grounded-cathode photomultiplier tube. Nucl Instrum Methods Phys Res Sect A 435(3):484-489

27. Sauer BE, Kara DM, Hudson JJ, Tarbutt MR, Hinds EA (2008) A robust floating nanoammeter. Rev Sci Instrum 79(12):126102

28. Carrera D, Manganini F, Boracchi G, Lanzarone E (2017) Defect detection in SEM images of nanofibrous materials. IEEE Trans Ind Inf 13(2):555-561

29. Ziabari M, Mottaghitalab V, McGovern S, Haghi A (2008) Measuring electrospun nanofibre diameter: a novel approach. Chin Phys Lett 25(8):3071

30. Milasius R, Malasauskiene J (2014) Evaluation of structure quality of web from electrospun nanofibres. Autex Res J 14(4):233-238 
31. Electrospinz SEM analyser, Electrospinz Ltd, Blenheim, New Zealand. www.electrospinz.co. $\mathrm{nz}$

32. Boracchi G, Carrera D, Wohlberg B (2014) Novelty detection in images by sparse representations. In: Proceedings of IEEE symposium on intelligent embedded systems, pp 47-54

33. Borrotti M, Lanzarone E, Manganini F, Ortelli S, Pievatolo A, Tonetti C (2017) Defect minimization and feature control in electrospinning through design of experiments. J Appl Polym Sci 134:44740

34. Coles SR, Jacobs DK, Meredith JO, Barker G, Clark AJ, Kirwan K, Stanger J, Tucker NJ (2010) A design of experiments (DoE) approach to material properties optimization of electrospun nanofibers. Appl Polym Sci 117(4):2251-2257

35. Box GEP, Draper NR (1987) Empirical model-building and response surfaces. In: Wiley Series in Probability and Statistics. Wiley, USA

36. Terkaj W, Tolio T (2019) The Italian flagship project: factories of the future. In: Tolio T, Copani G, Terkaj W (eds) Factories of the future. Springer

Open Access This book is licensed under the terms of the Creative Commons Attribution 4.0 International License (http://creativecommons.org/licenses/by/4.0/), which permits use, sharing, adaptation, distribution and reproduction in any medium or format, as long as you give appropriate credit to the original author(s) and the source, provide a link to the Creative Commons licence and indicate if changes were made.

The images or other third party material in this book are included in the book's Creative Commons licence, unless indicated otherwise in a credit line to the material. If material is not included in the book's Creative Commons licence and your intended use is not permitted by statutory regulation or exceeds the permitted use, you will need to obtain permission directly from the copyright holder. 